\title{
Experimental Method for Recording Epicardium Potentials and Cardiac Myocyte Shortening
}

\author{
Gustavo S Marchini ${ }^{1}$, Daniel S U Tamashiro ${ }^{2}$, Helena T Oyama ${ }^{3}$, Lucas Cortella ${ }^{3}$, Ismar N Cestari ${ }^{3}$, \\ Idágene A Cestari ${ }^{1,3}$ \\ ${ }^{1}$ Polytechnic School of University of São Paulo, São Paulo, Brazil \\ ${ }^{2}$ University of Campinas, São Paulo, Brazil \\ ${ }^{3}$ Bioengineering Division - Heart Institute (InCor), São Paulo, Brazil
}

\begin{abstract}
The hemodynamic changes observed in advanced stages of heart diseases are often accompanied by changes in the electrical and mechanical properties of cardiac myocytes. The objective of this work is to develop an experimental method for recording ventricle epicardium potentials in isolated rat hearts and isolated cardiac myocyte shortening. Briefly, rat heart was removed, aorta was cannulated and coronary arteries were retrogradely perfused with heated and oxygenated buffer solutions. Ag$\mathrm{AgCl}$ electrodes fixed in a silicone pouch placed around the heart were used to measure epicardium potentials. The perfusion was switched to an enzyme-containing solution for digestion of the heart and obtaining isolated cardiac myocytes. Measurements of shortening were made in cells electrically stimulated. The results suggest the possibility of relating the electrical behavior of the whole heart with mechanical properties of cardiac myocytes and may represent an useful tool in basic cardiac research.
\end{abstract}

\section{Introduction}

Cardiac excitation contraction coupling and mechanoelectric feedback are two processes that relate electrical and mechanical properties of the heart. In the former, electrical depolarization leads to mechanical contraction and, in the last, mechanical alterations can lead to changes in cardiac electrical activity [1].

Changes in both electrical and mechanical properties can occur in advanced stages of heart diseases. To understand and evaluate the effects at the whole heart and cellular scales two techniques may be used, respectively: epicardial potential mapping and isolated cardiac myocyte shortening. The first, allows the analysis of the spreading of action potentials on the epicardium surface and the velocities along and transverse to the fiber axis [2]. The second, gives assessment of cardiac contractility by quantify- ing contraction and relaxation kinetics (cross-bridge dynamics) allowing the evaluation of mechanical function [3].

This work describes an experimental method for evaluation of electrical and mechanical properties in a same experiment with the recording of ventricle epicardium potentials in isolated rat hearts and measuring of isolated cardiac myocyte shortening.

\section{Methods}

\subsection{Fabrication of electrodes}

Electrodes were fabricated manually using silver wire and a portable torch. From the diameter of the electrode it was possible to determine the length of the wire to be melted. Thus wire was slowly melted in the flame of the torch and a small electrode was formed. The silver electrodes were chlorided to form $\mathrm{Ag}-\mathrm{AgCl}$ that exhibit lower electrode-electrolyte impedance [4]. Six electrodes (0.5 $\mathrm{mm}^{2}$ surface area; $0.8 \mathrm{~mm}$ interelectrode spacing) were fixed in a silicone pouch to maintain contact with the heart epicardial surface.

\subsection{Animal preparation and heart cannu- lation}

Experiments were performed in six-week-old male Wistar rats and were carried out in accordance with the Committee of Ethics in the Use of Animals of School of Medicine, University of São Paulo. Rat was heparinized $(5000 \mathrm{U} / \mathrm{kg})$ and, after 30 minutes, anesthetized with sodium thiopental and euthanized by cervical dislocation. Hearts were then excised and placed in ice-cold, oxygenated buffer solution containing (in $\mathrm{mmol} / \mathrm{l}$ ): 134 $\mathrm{NaCl}, 4.0 \mathrm{KCl}, 1.2 \mathrm{NaH}_{2} \mathrm{PO}_{4}, 10$ HEPES, $0.5 \mathrm{MgSO}_{4}$, $1.25 \mathrm{CaCl}_{2}$ and $11 \mathrm{D}$-glucose (pH 7.4) [5]. 


\subsection{Measuring of epicardium potentials}

The heart was mounted on a Langendorff perfusion system with constant flow (Fig. 1) and perfused with the same buffer solution at $37{ }^{\circ} \mathrm{C}(6 \mathrm{ml} / \mathrm{min})$. The silicone pouch with the electrodes enveloped the heart and a single reference electrode was placed at the aorta (Fig. 2). Unipolar epicardial potentials were analog filtered (bandpass 0.1 to $300 \mathrm{~Hz}$ ) and amplified (Gould Electronics, Chandler, AZ, USA), digitalized with a sampling frequency of $8 \mathrm{kHz}$ (DI720, DataQ Instruments, Akron, OH, USA) and stored in a computer for post-processing. A MATLAB (MathWorks, Inc., Nattick, MA, USA) algorithm was used to determine time delays between epicardium potentials based on the minimum values of the time derivative of shortening.

\subsection{Cardiac myocyte isolation}

The perfusion solution was switched to the same buffer without $\mathrm{Ca}^{2+}$ for 4 minutes followed by digestion step performed with perfusion buffer containing collagenase type II $(100 \mathrm{U} / \mathrm{ml}$; Worthington Biochemical, Lakewood, NJ, USA) and $20 \mu \mathrm{M} \mathrm{Ca}^{2+}$ until the heart became pale and flaccid (20-30 min)(Fig. 3A). The ventricles were separated from the heart, cut into small pieces and gently tritured with a pipette in the buffer solution with $50 \mu \mathrm{M} \mathrm{Ca}^{2+}$ and 1\% BSA (Sigma, St Louis, MO, USA). Following filtration through $200 \mu \mathrm{m}$ nylon mesh and sedimentation, the cell pellet was washed four times in buffer solution plus $1 \%$ BSA with gradually increasing of $\left[\mathrm{Ca}^{2+}\right](0.1,0.2,0.5$ and $1 \mathrm{mM}$ ). Fig. 3B shows an example of isolated cardiac myocytes obtained by enzymatic dissociation.

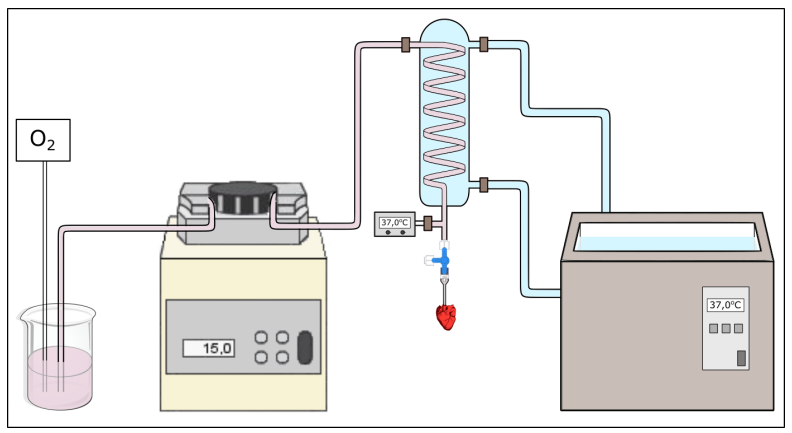

Figure 1. Schematic depiction of the Langendorff perfusion system with constant flow. A peristaltic pump drives the oxygenated solution through a heat exchanger coil connected to a water bath. Heart is cannulated by the aorta allowing retrograde perfusion through coronary arteries.

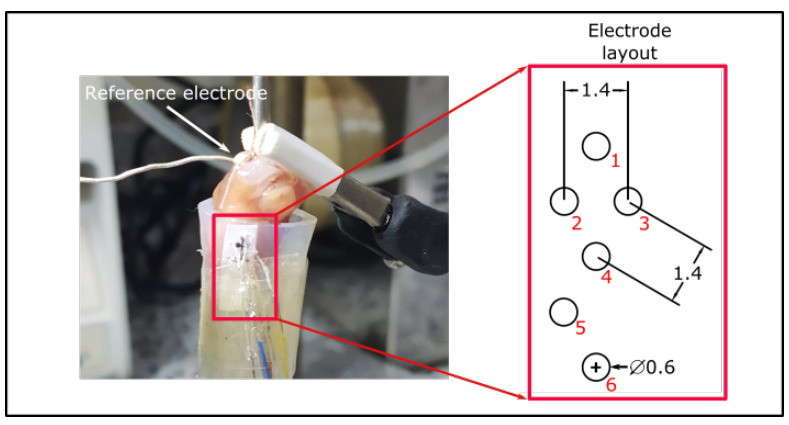

Figure 2. On left, image of the silicone pouch with the electrodes; on right, the electrode layout with the dimensions given in $\mathrm{mm}$. The red numbers are the identification of electrodes.
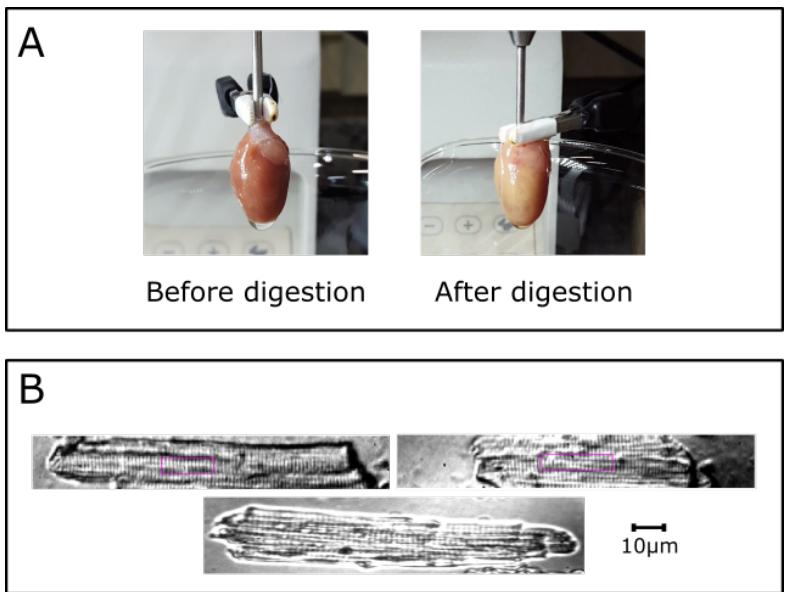

Figure 3. A: on left, cannulated heart just before the start of perfusion with collagenase; on right, the same heart after enzymatic digestion. B: isolated cardiac myocytes obtained by enzymatic digestion.

\subsection{Measurement of cardiac myocyte short- ening}

Shortening of cardiac myocytes by electrical field stimulation $(1 \mathrm{~Hz})$ was recorded using a video edge detection system composed by an inverted microscope (Eclipse TS100; Nikon, Tokyo, Japan) with an analog camera (Myocam; IonOptix, Milton, MA, USA). Contraction signals were analyzed using commercially data analysis software (IonWizard; IonOptix, Milton, MA, USA) providing typical contraction parameters including resting cell length, percentage cell shortening (i.e., percentage of resting cell length), shortening and relaxation velocities and time intervals to reach the peak of contraction and 50\% resting cell length. The parameters were calculated with the mean of following contractions of a cardiac myocyte. All experiments were conducted at room temperature $\left(23^{\circ} \mathrm{C}\right)$. 

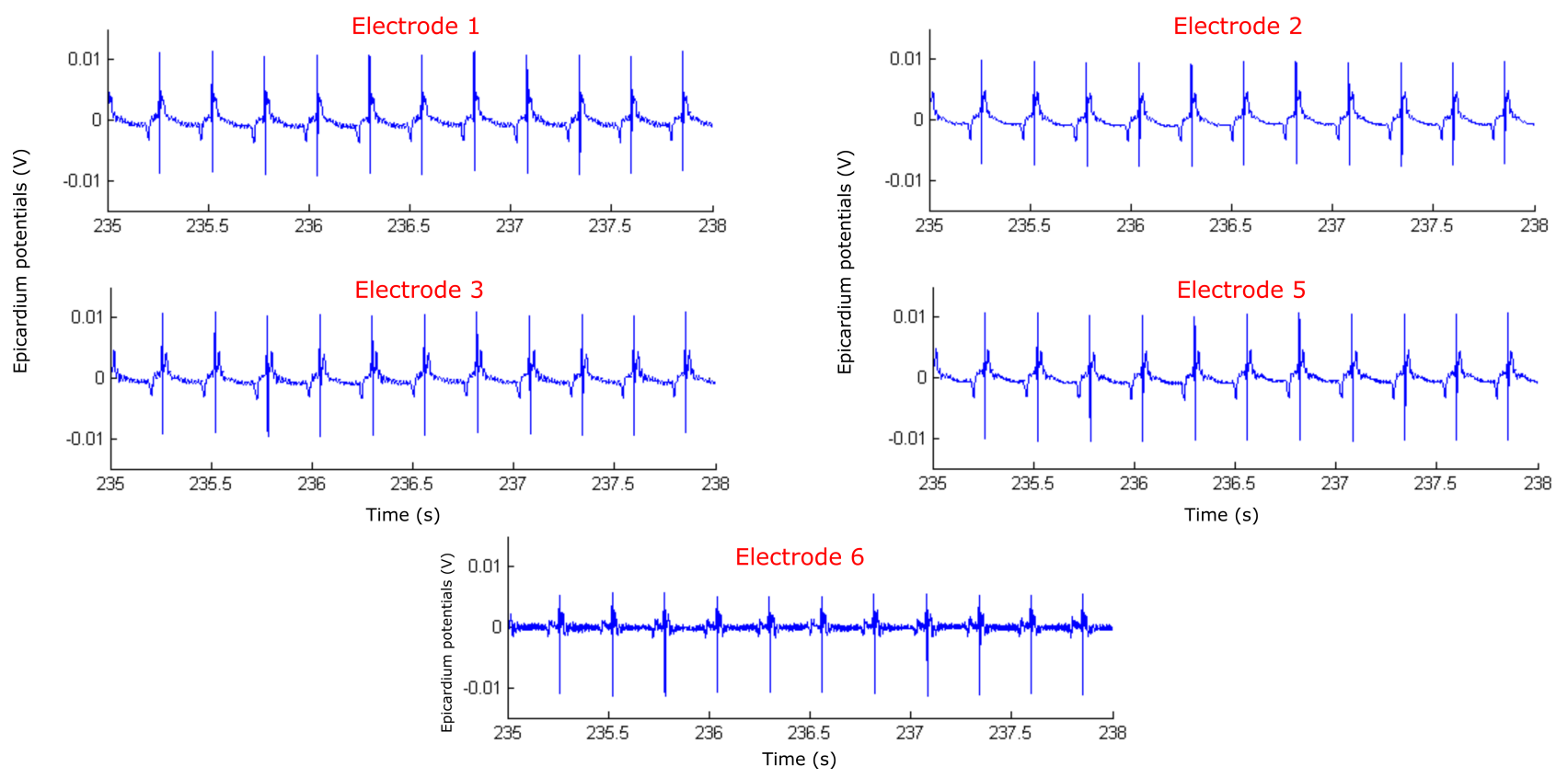

Figure 4. Signals of ventricle epicardium potentials in sinus rhythm recorded in five electrodes in one experiment.

Table 1. Delay values between the epicardium potentials of different electrodes for three experiments. The time intervals correspond to the delay between the most advanced signal and the signaling identified on the respective electrode (fig. 1B).

\begin{tabular}{cccccc}
\hline \hline \multicolumn{2}{c}{ Experiment $1(\mathrm{n}=27)$} & \multicolumn{2}{c}{ Experiment $2(\mathrm{n}=15)$} & \multicolumn{2}{c}{ Experiment $3(\mathrm{n}=81)$} \\
\hline Electrode & Mean \pm SD $(\mathrm{ms})$ & Electrode & Mean \pm SD $(\mathrm{ms})$ & Electrode & Mean \pm SD $(\mathrm{ms})$ \\
\hline 6 & - & 6 & - & 3 & - \\
5 & $0.54 \pm 0.08$ & 5 & $0.58 \pm 0.06$ & 2 & $0.32 \pm 0.07$ \\
1 & $0.69 \pm 0.06$ & 3 & $1.50 \pm 0.14$ & 6 & $0.59 \pm 0.07$ \\
3 & $0.82 \pm 0.06$ & 4 & $1.63 \pm 0.12$ & 4 & $0.61 \pm 0.07$ \\
2 & $1.20 \pm 0.08$ & 2 & $2.02 \pm 0.08$ & 5 & $0.78 \pm 0.11$ \\
\hline \hline
\end{tabular}

\section{Results}

\subsection{Epicardium potentials}

Fig. 4 shows an example of ventricle epicardium potentials in sinus rhythm for one experiment. Table 1 shows the time delays obtained in the three experiments. Mean and standard deviation were calculated on time delays obtained from the same epicardium potential on different electrodes. Some electrodes were omitted from the analysis because they did not show signal, probably due to poor contact between electrode and epicardium.

\subsection{Cardiac myocyte shortening}

Fig. 5 presents an example of a result of cardiac myocyte shortening. The top of the figure shows shortening of an electrically stimulated isolated myocyte and the bottom shows the average shortening. Table 2 summarizes mean and standard deviation of contraction parameters calculated in isolated cardiac myocytes $(n=36)$.

\section{Discussion}

The presented method shows the availability of recording ventricle epicardium potentials in Langendorff perfused rat hearts and isolated cardiac myocyte shortening. For epicardium potentials, time delays were used to show one possible analysis for the investigation of whole heart electrophysiology. The analysis can be extended with the increase in the electrode density [6] and interpolation of the signals for a better reconstruction of the electrical activity on the epicardial surface [7]. Different maps for analysis like isochronal, isopotential and phase maps, should be considered as well as the adoption of new structures that improve the contact between the electrodes and the surface of the heart [8]. 

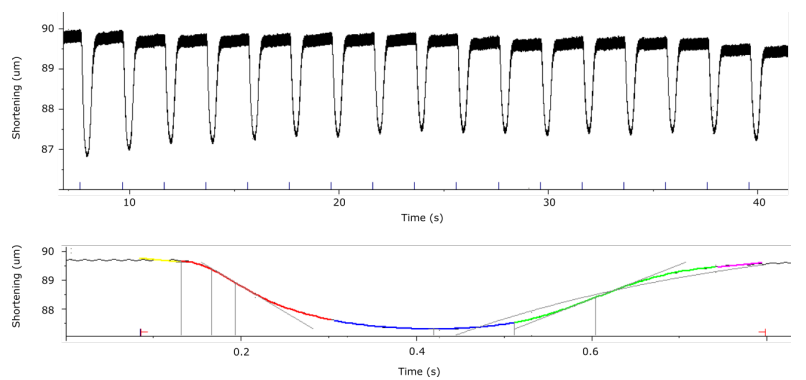

Figure 5. Shortening signal of an electrically stimulated isolated cardiac myocyte (top). Average shortening (bottom). The regions in yellow, red, blue, green and purple are used to calculate, respectively, cell length, shortening velocity, percentage of shortening (and time to peak), relaxation velocity and time to $50 \%$ relaxation.

Table 2. Contraction parameters measured in electrically stimulated isolated cardiac myocytes $(n=36)$.

\begin{tabular}{lcc}
\hline \hline & Mean & SD \\
\hline Cell length $[\mu \mathrm{m}]$ & 87,850 & 19,846 \\
Percentage of shortening & 3,053 & 1,387 \\
Shortening velocity $[\mu \mathrm{m} / \mathrm{s}]$ & $-29,722$ & 21,424 \\
Relaxation velocity $[\mu \mathrm{m} / \mathrm{s}]$ & 20,612 & 16,005 \\
Time to peak $[\mathrm{s}]$ & 0,207 & 0,043 \\
Time to 50\% relaxation $[\mathrm{s}]$ & 0,346 & 0,088 \\
\hline \hline
\end{tabular}

For the isolated cardiac myocyte shortening, the measurements were conducted at room temperature. However, the temperature influences contraction parameters such as time to peak and time to $50 \%$ relaxation and, also, reduces the biological variability [9] and its control should be adopted in future experiments. Measurements of intracellular calcium can be considered to complement the analysis of contractility of isolated cardiac myocytes.

\section{Conclusion}

The results obtained in the experiments suggest the possibility of relating the electrical behavior of the whole heart with isolated cardiac myocytes mechanical properties and may represent an useful tool in basic cardiac research.

\section{Acknowledgements}

This work was supported by São Paulo Research Foundation (FAPESP) grant 2013/24543-3.

\section{References}

[1] Pfeiffer ER, Tangney JR, Omens JH, McCulloch AD. Biomechanics of Cardiac Electromechanical Coupling and Mechanoelectric Feedback. Journal of Biomechanical Engineering 2014;136(2):0210071-02100711.

[2] Dhein S, Mohr FW, Delmar M. Practical Methods in Cardiovascular Research. Springer Science \& Business Media, 2005. ISBN 978-3-540-40763-8.

[3] Brady AJ. Mechanical properties of isolated cardiac myocytes. Physiological reviews 1991;71(2):413-428.

[4] Geddes LA, Baker LE, Moore AG. Optimum electrolytic chloriding of silver electrodes. Medical and biological engineering 1969;7(1):49-56.

[5] Louch WE, Sheehan KA, Wolska BM. Methods in Cardiomyocyte Isolation, Culture, and Gene Transfer. Journal of molecular and cellular cardiology 2011;51(3):288-298.

[6] Macchi E, Cavalieri M, Stilli D, Musso E, Baruffi S, Olivetti G, Ershler PR, Lux RL, Taccardi B. High-density epicardial mapping during current injection and ventricular activation in rat hearts. American Journal of Physiology Heart and Circulatory Physiology 1998;275(5):H1886-H1897.

[7] Lu W, Yang C, Fang Z, Liu X, Zhu X, Wei D. Implementation of a novel interpolating method to epicardial potential mapping for atrial fibrillation study. Computers in Biology and Medicine 2010;40(4):456-463.

[8] Xu L, Gutbrod SR, Bonifas AP, Su Y, Sulkin MS, Lu N, Chung HJ, Jang KI, Liu Z, Ying M, Lu C, Webb RC, Kim JS, Laughner JI, Cheng H, Liu Y, Ameen A, Jeong JW, Kim GT, Huang Y, Efimov IR, Rogers JA. 3D multifunctional integumentary membranes for spatiotemporal cardiac measurements and stimulation across the entire epicardium. Nature communications 2014;5:3329.

[9] Chung CS, Campbell KS. Temperature and transmural region influence functional measurements in unloaded left ventricular cardiomyocytes. Physiological Reports 2013;1(6).

Address for correspondence:

Gustavo Shimabukuro Marchini

University of São Paulo

Bioengineering Division - Heart Institute (InCor)

Av. Dr. Enéas de Carvalho Aguiar, 44

So Paulo, SP - 05403-900

gustavo.marchini@usp.br 